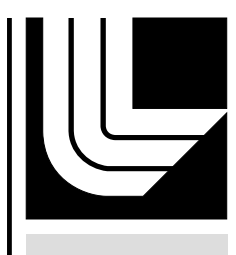

LAW RENCE LIVERMORE N A TION A L LABORATORY

\title{
UCRL-TR-207260
}

\section{APS Performance Summary - Update}

J. Gross, B. Kozioziemski, A. Waters, H. Martz

October 15, 2004 
This document was prepared as an account of work sponsored by an agency of the United States Government. Neither the United States Government nor the University of California nor any of their employees, makes any warranty, express or implied, or assumes any legal liability or responsibility for the accuracy, completeness, or usefulness of any information, apparatus, product, or process disclosed, or represents that its use would not infringe privately owned rights. Reference herein to any specific commercial product, process, or service by trade name, trademark, manufacturer, or otherwise, does not necessarily constitute or imply its endorsement, recommendation, or favoring by the United States Government or the University of California. The views and opinions of authors expressed herein do not necessarily state or reflect those of the United States Government or the University of California, and shall not be used for advertising or product endorsement purposes.

This work was performed under the auspices of the U.S. Department of Energy by University of California, Lawrence Livermore National Laboratory under Contract W-7405-Eng-48. 


\title{
APS Performance Summary - Update ${ }^{1}$
}

\author{
Jeff Gross, Bernard Kozioziemski, Amy Waters, and Harry Martz \\ Lawrence Livermore National Laboratory, Livermore, CA
}

\section{Introduction}

High Energy Density Physics (HEDP) Experiments play an important role in corroborating the improved physics codes that underlie LLNL's Stockpile Stewardship mission. Conducting these experiments, whether on the National Ignition Facility (NIF) or another national facility such as Omega, will require not only improvement in the diagnostics for measuring the experiment, but also detailed knowledge of the as-built target components and assemblies themselves. To assist in this effort, a defined set of well-known reference standards have been built and are being used to quantify the performance of different characterization techniques. Without the critical step of using reference standards for qualifying characterization tools there can be no verification of either commercial or internally-developed characterization techniques and thus there is an uncertainty in the input to the as-built physics codes [Waters et. al. 2004].

In FY03, two reference standards were fabricated and characterized using metrology tools. One of the reference standards was built with a cylindrical geometry and the second reference standard was built with a spherical geometry. The standards were designed for manufacturability, stability, and to provide a range of features that can be measured using NDE methods. Detailed information about these methods has been previously documented [Hibbard, et al. 2003].

\section{Advanced Photon Source (APS)}

The performance of different DR/CT systems for mesoscale imaging has been benchmarked. Several x-ray systems have been characterized and documented to date, including two microfocus systems [Waters, et al. 2004; Waters, et al. 2004a] and one synchrotron based system [Brown, et al. 2004]. A synchrotron source used was the Advance Photon Source (APS) located at Argonne National Laboratory. Synchrotron based systems have several advantages over standard microfocus systems, including very high flux, and the capability to tune energies. However, because synchrotrons are large and expensive to run, reserving time on a beamline can be extremely difficult, if not impossible. System characterization experiments were therefore executed at APS in conjunction with existing ICF project events.

\footnotetext{
${ }^{1}$ This work was performed under the auspices of the U. S. Department of Energy by the University of California Lawrence Livermore National Laboratory under contract No. W-7405-Eng-48.
} 
Imaging experiments were carried out using the APS 1-ID beam-line at Argonne National Laboratory. The 1-ID beam line uses an undulator insertion device to produce photons. A silicon monochrometer in the beam path was set for $10-\mathrm{keV}$ photons with a $2-\mathrm{eV}$ bandwidth. The x-ray beam is not spatially uniform due to the radiation pattern produced by the undulator magnets. Further, the structure has a varying temporal component. The $\mathrm{x}$-ray beam had a flux of $10^{11}$ photons/second in a 2-mm x 2-mm beam with a 30-microradian divergence. The beam spot size in horizontal and vertical directions was 15 and $250 \mu \mathrm{m}$, respectively. A 300- $\mu \mathrm{m}$ thick cadmium tungstate $\left(\mathrm{CdWO}_{4}\right)$ scintillator was optically magnified with a Mitutoyo 10x objective lens and imaged onto a Princeton Instruments liquid-nitrogen cooled 1024x1024 16-bit CCD camera. The detector system was positioned at varying distances behind the object. The APS source is located 60 meters from the object so the beam can be considered parallel and the effect of magnification is negligible. Thus the effective pixel size at the object was $2 \mu \mathrm{m}$. Figure 1 shows the experimental design.

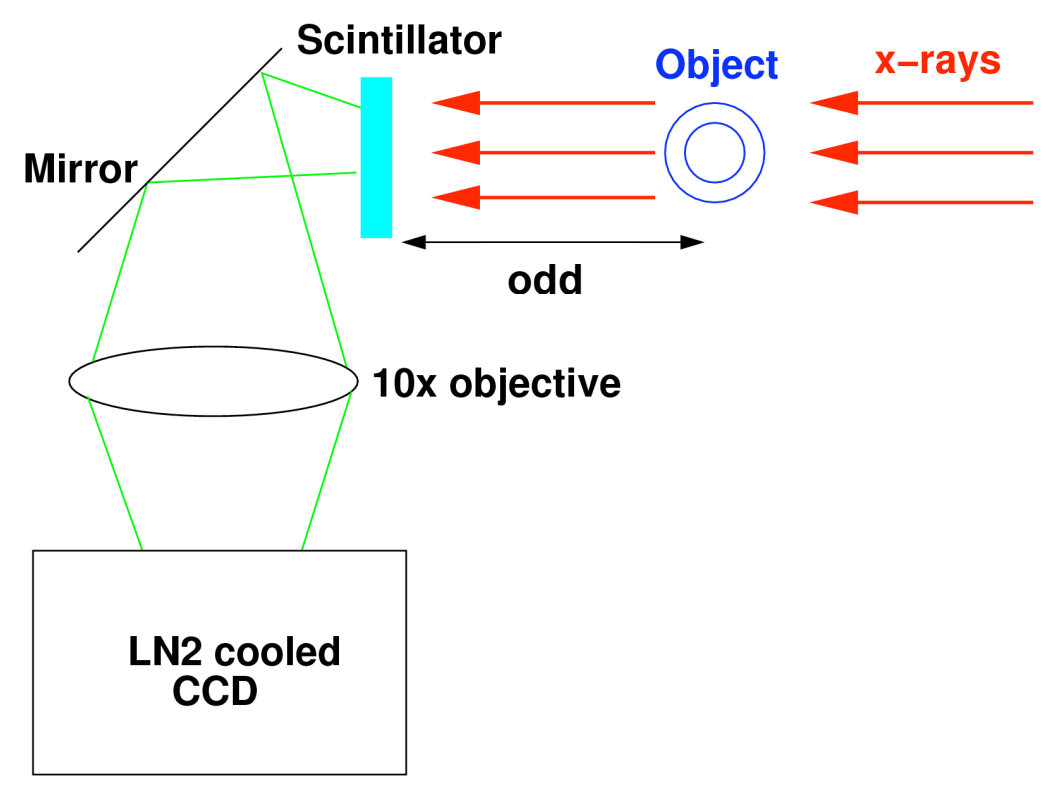

Figure 1. The experimental setup used at the APS 1-ID beam line. Monochromatic x-rays pass through the object and are collected in the $\mathrm{CdWO}_{4}$ scintillator placed a distance odd from the object. A $10 \mathrm{x}$ microscope objective lens couples the scintillator to the CCD.

The object and detector were both positioned on translation stages to enable alignment within the beam. A polished thin $(0.51 \mathrm{~mm})$ tantalum foil edge was imaged with objectto-detector distances (ODD) of 262, 462 and $1162 \mathrm{~mm}$. The ODD distances used for imaging the spherical reference standard were 62,112 and $262 \mathrm{~mm}$, respectively.

The beam line was configured for phase-enhanced imaging rather than full tomography, however the object rotation stage enabled a series of different angles to be imaged. Steps of 5 degrees were taken over 180 degrees. One image at each angle was taken due to the large number of images. Object walk-off during rotation necessitated translation of the reference standard between rotation steps. Because of the difficulty in trying to register all of the images, they were not reconstructed into CT slices. 


\section{Digital radiography system performance}

The APS digital radiography system performance was characterized with the same tantalum edge and analysis procedures used to characterize other mesoscale DR/CT imaging systems. The results from other systems are documented in previous reports. [Waters, et al. 2004; Waters, et al. 2004a; Brown, et al. 2004]

Sets of measurements for the Ta edge were taken at each of the three object-to-detector distances. Each set of measurements consisted of two incident intensity $\left(\mathrm{I}_{0}\right)$ images and two images with the edge $\left(I_{E}\right)$. Two images were acquired in each measurement set in an effort to average out the time-varying structure of the beam. Average images of the edge and $\mathrm{I}_{0}$ were created by adding the two images of either $\mathrm{I}_{\mathrm{E}}$ or $\mathrm{I}_{0}$ and dividing by two. The dark current for the CCD was about 950 counts and was not taken into account as no image was acquired. While taking the dark current into account may tend to improve the MTF by accounting for hardware anomalies, it is likely a negligible affect.

Transmission $\left(\mathrm{I} / \mathrm{I}_{\mathrm{o}}\right)$ images were created for both single and averaged images and lineout and MTF measurements were determined for each. Lineout results from both the single and averaged images corresponding to three object-to-detector distances are shown in Figures 2 and 3 respectively.

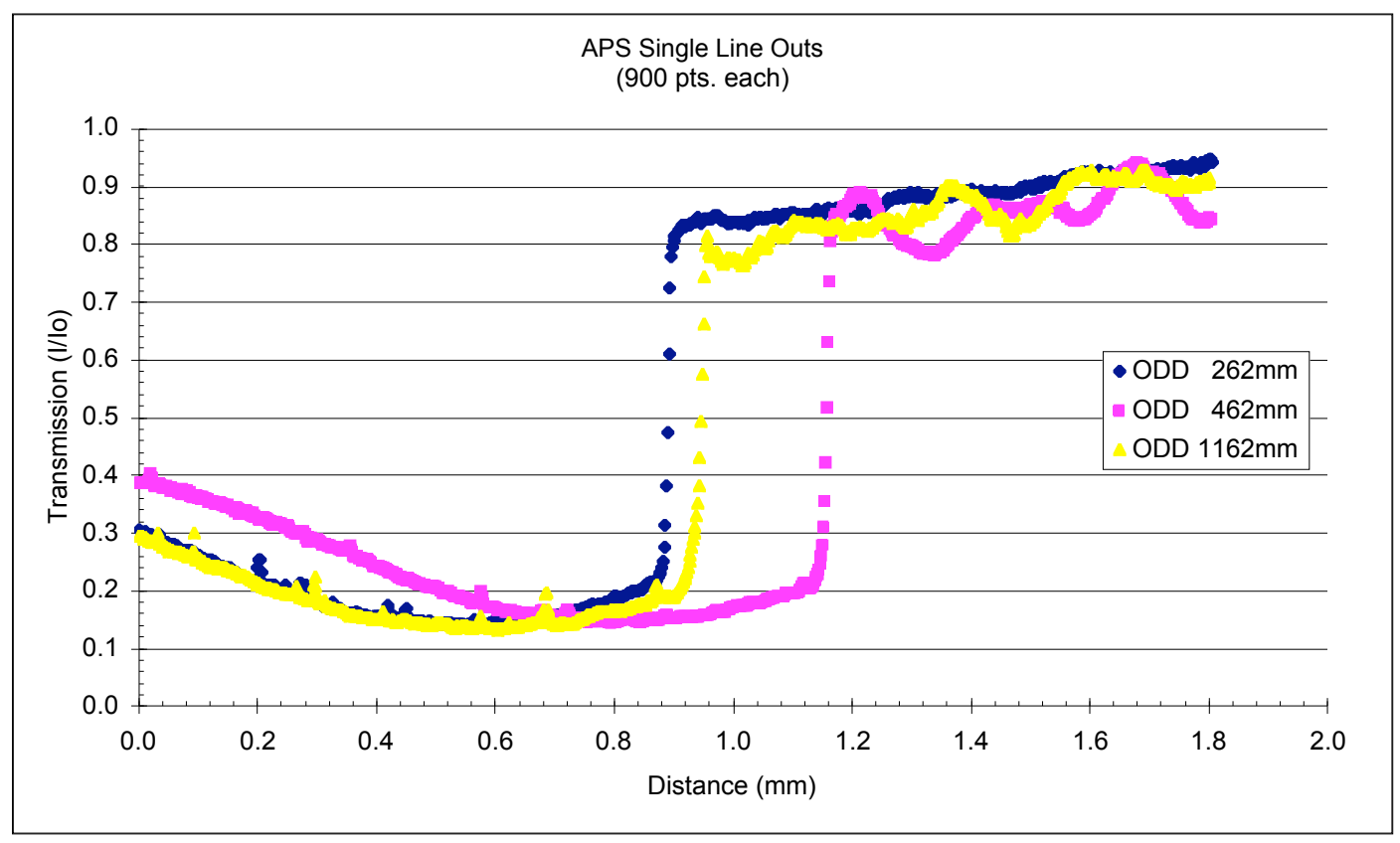

Figure 2. Three 10-pixel wide one-dimensional lineouts taken from the individual images of the single thin $(0.51 \mathrm{~mm})$ tantalum edge transmission images at three object-to-detector distances. Note the wave structure in the lineout away from the edge and under the edge the lineout is not flat. The wave structure is due to the temporal fluctuations in the synchrotron source. 


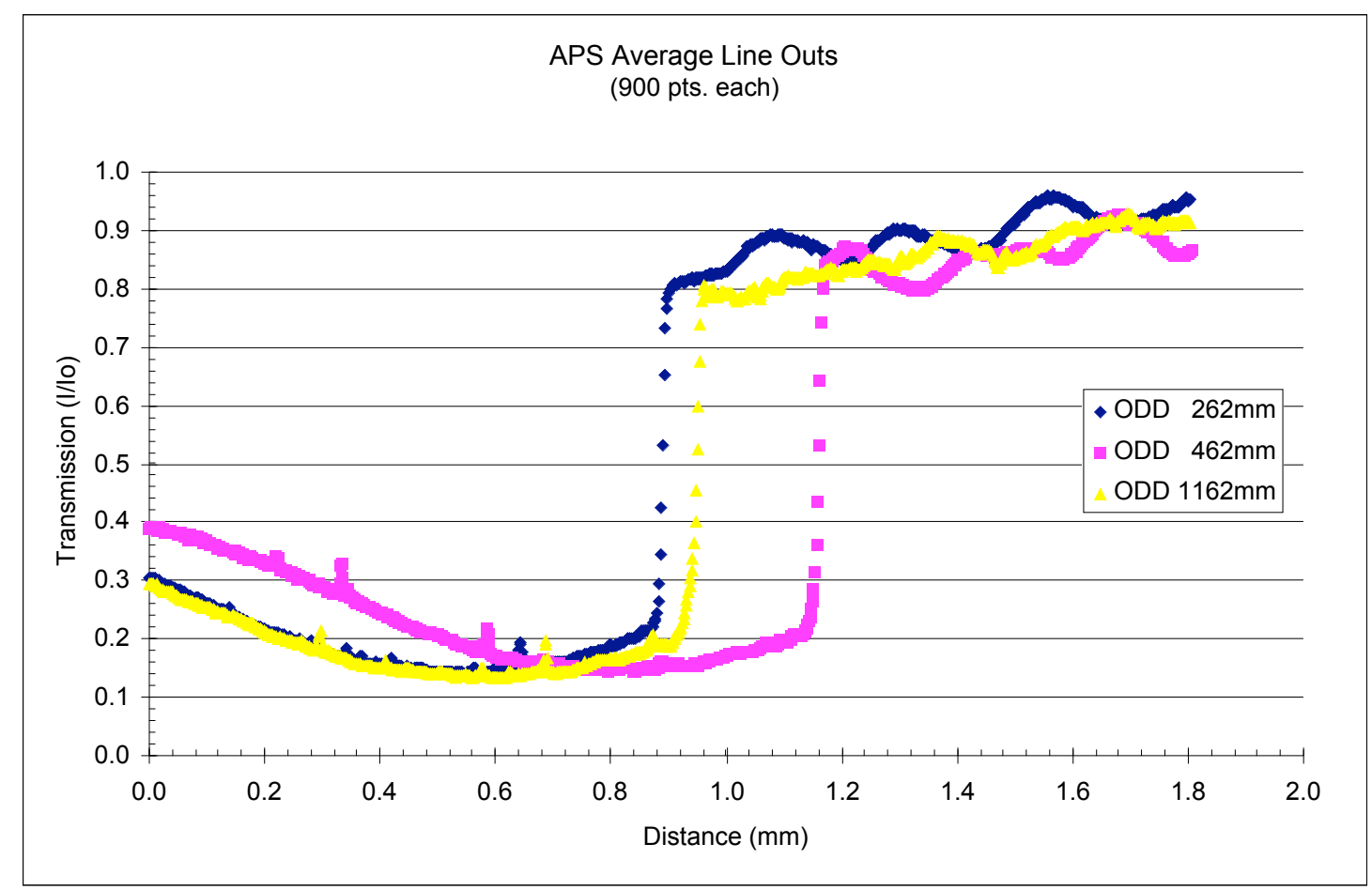

Figure 3. Three 10-pixel wide one-dimensional lineouts taken from the averaged tantalum edge transmission images at three object-to-detector distances. The averaging of the images does not effectively remove the temporal effects of the beam, and in the $262 \mathrm{~mm}$ data set appears to amplify the effect.

The DR MTF's for the APS data were determined from the lineouts from both single and averaged transmission data sets and are shown in Figures 4 and 5, respectively. To remain consistent with earlier reports the data is presented unsmoothed.

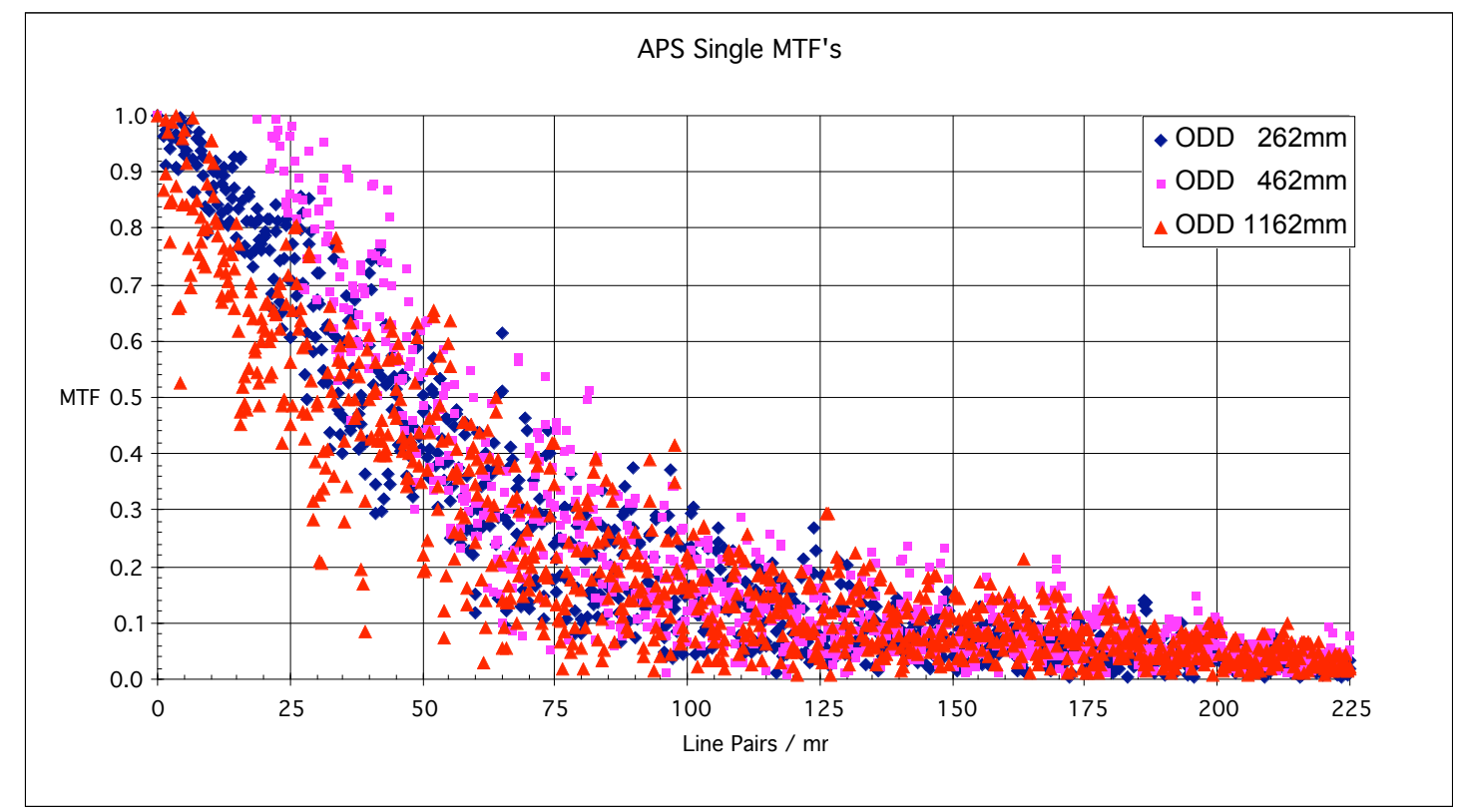

Figure 4. DR MTFs derived from the single images of the Ta edge using the lineouts in Figure 2. 


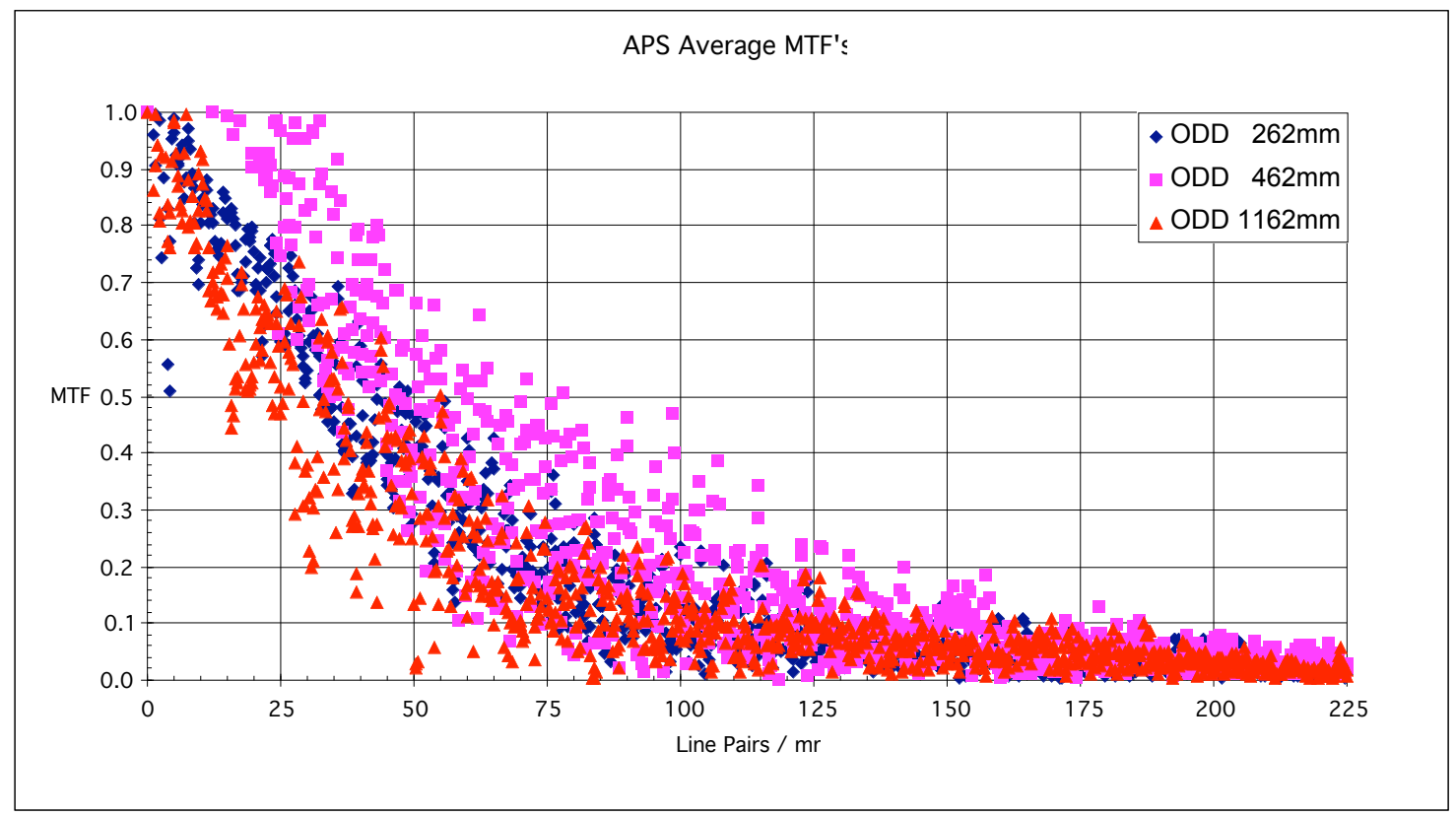

Figure 5. DR MTFs derived from the averaged images of the Ta edge using the lineouts in Figure 3.

The signal to noise ratio (SNR) was also measured for the APS system. The SNR is defined as the difference between the mean of two signals (in our case the two signals are within, $S_{2}$, and outside, $S_{1}$, the Ta edge) divided by the square root of the sum of the squares of the standard deviation:

$$
\frac{S_{1}-S_{2}}{\sqrt{\sigma_{1}^{2}+\sigma_{2}^{2}}},
$$

where $S$ is the mean of the signal and $\sigma$ is the standard deviation of the signal. Three two-dimensional SNR measurements were made using one averaged image of the Ta edge at an ODD of $262 \mathrm{~mm}$ and are presented in Table 1.

Table 1. SNR measurements from one averaged transmission image of the Ta edge at odd of $262 \mathrm{~mm}$.

\begin{tabular}{|c|c|c|}
\hline Area (pixels) & SNR & Average SNR \\
\hline $147 \times 149$ & 15.7 & \multirow{2}{*}{$16.15 \pm 5.4$} \\
\hline $148 \times 151$ & 21.7 & \\
\hline $147 \times 151$ & 11.0 & \\
\hline
\end{tabular}




\section{Spherical reference standard}

Several projection images of the reference standard were acquired. One image is presented in Figure 6 as percent transmission $\left[\left(\mathrm{I} / \mathrm{I}_{0}\right) \times 100\right]$. X-ray attenuation is a function of x-ray energy, path length, material density and elemental composition. In the image shown below, dark regions indicate either longer path lengths, or higher density of material. Conversely, lighter regions indicate less density of material or shorter path lengths.

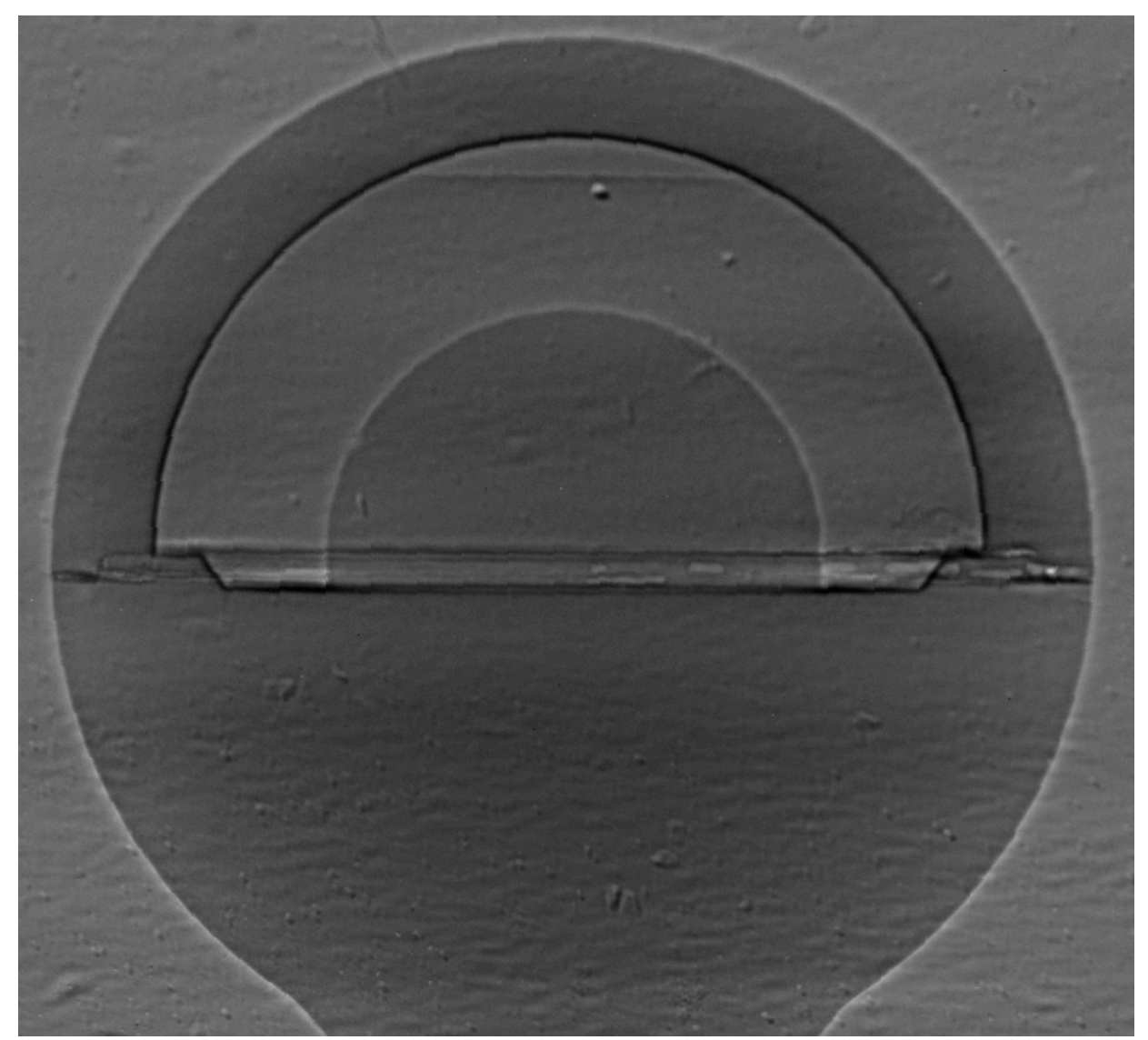

Figure 6. Percent transmission image of the spherical reference standard acquired at APS. In this image, darker regions may indicate longer path lengths, or greater material density, while light areas may indicate shorter path lengths, or less dense material

Note the area around the step joint in the Figure 6. It appears there are unbonded regions in the joint, appearing as lighter areas. In addition, there appears to be excess material, probably glue, seen above the step joint on the right side of the image. Note the gap at the top of the Carbonized Resocinol Formaldehyde (CRF) foam that is easily seen. The brightness around the outer edge of the reference standard is due to phase, as explained below. Similarly, the very dark edges on the inner wall are also due to phase effects. 


\section{Phase Contrast Imaging}

Contrast at interfaces is enhanced due to the discontinuous change in refractive index [Silkins, et al. 1996; Gureyev, et al. 1999; Margaritondo, et al. 1999]. X-rays passing near the interface are deflected only tens of micro-radians. However, by placing the detector far from the object, the slight deflection becomes, to first order, a detectable linear displacement. This shows up as a light-to-dark transition at interfaces in the image. The edge contrast increases as the object-to-detector distance is increased (see Figure 6). Figure 7 shows two images taken at different object-to-detector distances. The phaseenhanced images show increased contrast with increased object-to-detector distance. The interfaces and features appear to have higher contrast in the image taken at the larger object-to-detector distance. Features, such as the air-CRF interface, the glue, and joints are easier to locate in the image with ODD of $262 \mathrm{~mm}$ shown in Figure 7. A lineout extending across the inner $\mathrm{CH}(\mathrm{Br})$ interface through the edge of the $\mathrm{CRF}$ is shown in Figure 8. The contrast at the CRF-air interface is increased at the larger object-detector distance. By using phase contrast effects, features with low absorption contrast can be imaged.

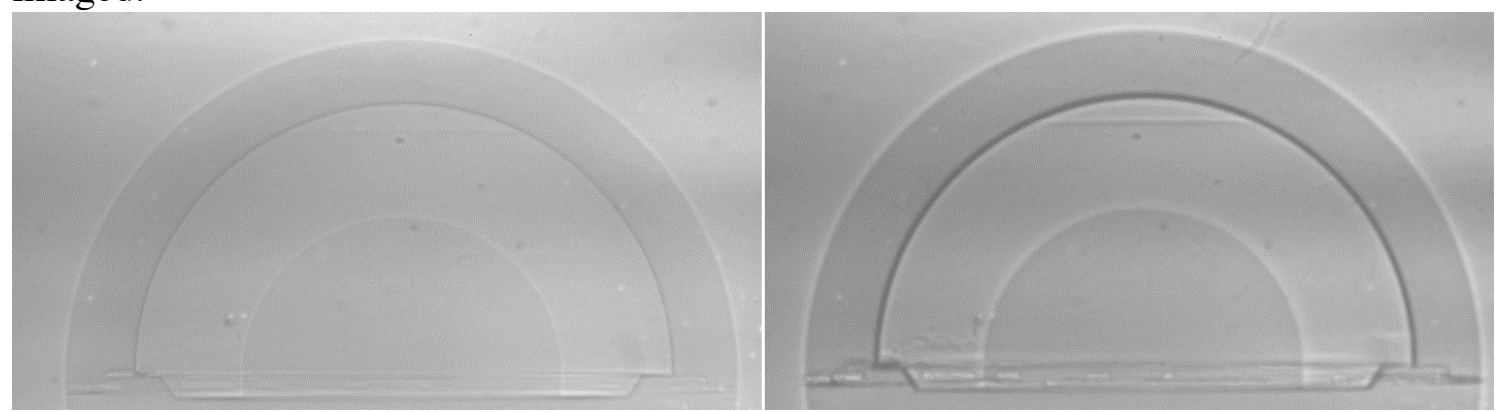

Figure 7. Images of the reference standard taken with object-detector distance left: $62 \mathrm{~mm}$ and right: 262 $\mathrm{mm}$. The interfaces and features appear to have higher contrast in the right image due to phase effects.

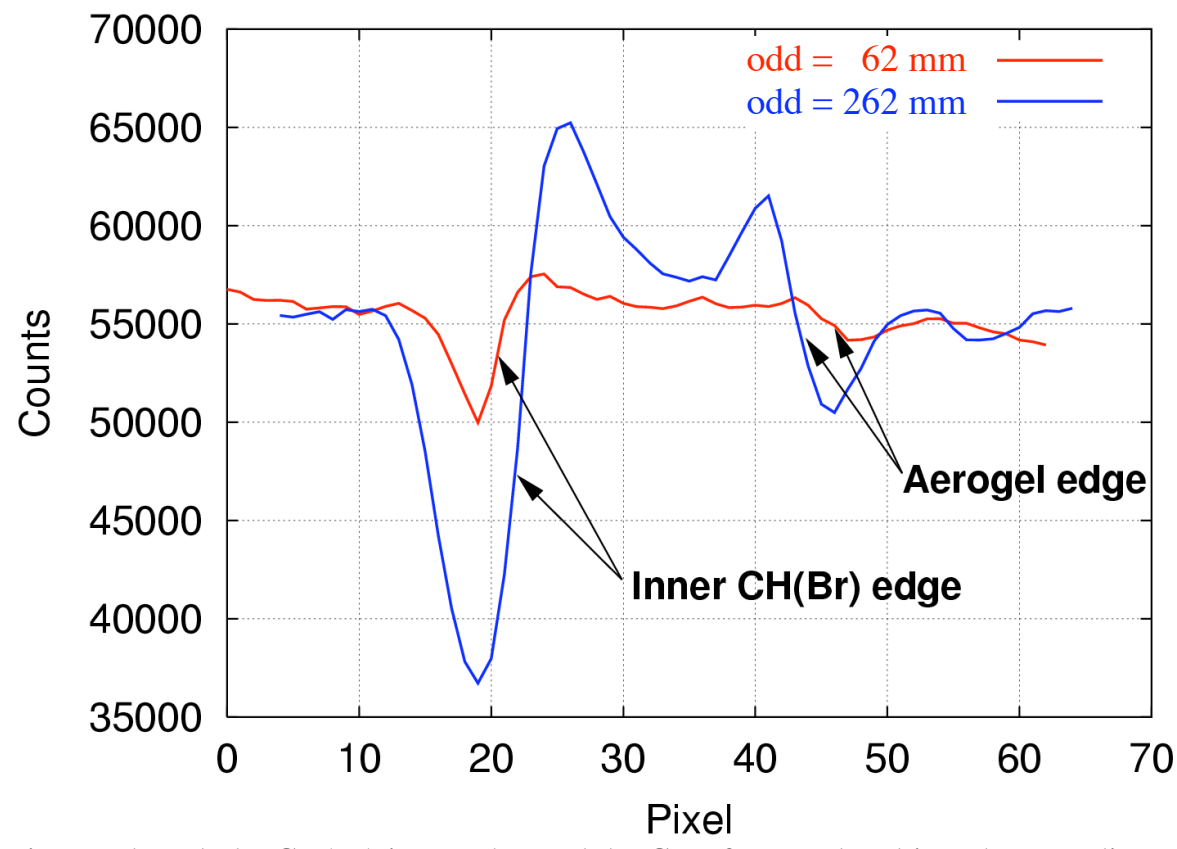

Figure 8. Lineout though the $\mathrm{CH}(\mathrm{Br})$ inner edge and the $\mathrm{CRF}$ foam. The object-detector distance, odd, was increased from $62 \mathrm{~mm}$ to $262 \mathrm{~mm}$. The contrast across the interfaces increases significantly with odd. 


\section{Summary}

The measurement of digital radiography and computed tomography system performance parameters for several systems is in progress. In this report we have documented the DR MTF and SNR measurements for the APS Beamline 1-ID. Several images of the spherical reference standard were acquired, and phase contrast effects in the images were shown to increase with object-to-detector distance.

\section{Future work}

- Measure DR MTFs with new edge reference standards;

- Configure staging at beamline to facilitate fast and easy collection of CT data;

- Develop quantitative data analysis techniques for 3-D CT data;

- Measure CT MTF system performance tube test objects;

- Compare the quantitative systems performance characteristics with other systems, including synchrotron and other commercially available and LLNL developed DR/CT systems.

\section{References}

Brown, W., A. Waters, and H. Martz, “ALS Performance Summary - Update”, Lawrence Livermore National Laboratory, CA, UCRL-TR-XXXX (2004)

Hibbard, R., M. Bono, A. Waters, and H. Martz, "TechBase Final Report for: Production and Documentation of Mesoscale Metrology and Characterization Reference Standards", Lawrence Livermore National Laboratory, Livermore, CA, UCRL-ID-XXXX (2004).

Waters, A., H. Martz, C. Logan, J. Gross and D. Chinn, "KCAT Performance Summary - Update, Rev. 1", Lawrence Livermore National Laboratory, Livermore, CA, UCRLTR-203519 (2004).

Waters, A., H. Martz, C. Logan, J. Gross and D. Chinn, "Xradia Performance Summary Update", Lawrence Livermore National Laboratory, Livermore, CA, UCRL-TR-206565 (2004a). 